University of Nebraska - Lincoln

DigitalCommons@University of Nebraska - Lincoln

\title{
The effect of defects on the electronic structure of long chain ferroelectric polymers
}

Jie Xiao

University of Nebraska-Lincoln, jie.xiao@helmholtz-berlin.de

Xin Zhou

Pennsylvania State University

Q. M. Zhang

Pennsylvania State University

Peter A. Dowben

University of Nebraska-Lincoln, pdowben@unl.edu

Follow this and additional works at: https://digitalcommons.unl.edu/physicsdowben

Part of the Physics Commons

Xiao, Jie; Zhou, Xin; Zhang, Q. M.; and Dowben, Peter A., "The effect of defects on the electronic structure of long chain ferroelectric polymers" (2009). Peter Dowben Publications. 240.

https://digitalcommons.unl.edu/physicsdowben/240

This Article is brought to you for free and open access by the Research Papers in Physics and Astronomy at DigitalCommons@University of Nebraska - Lincoln. It has been accepted for inclusion in Peter Dowben Publications by an authorized administrator of DigitalCommons@University of Nebraska - Lincoln. 


\title{
The effect of defects on the electronic structure of long chain ferroelectric polymers
}

\author{
Jie Xiao, ${ }^{1}$ Xin Zhou, ${ }^{2}$ Q. M. Zhang, ${ }^{2}$ and P. A. Dowben ${ }^{1, a)}$ \\ ${ }^{1}$ Department of Physics and Astronomy, Nebraska Center for Materials and Nanoscience, \\ University of Nebraska-Lincoln, Lincoln, Nebraska 68588-0111, USA \\ ${ }^{2}$ Department of Electrical Engineering, Pennsylvania State University, University Park, \\ Pennsylvania 16802, USA
}

(Received 23 March 2009; accepted 18 July 2009; published online 26 August 2009)

\begin{abstract}
The apparent molecular density of states from combined photoemission and inverse photoemission spectroscopies is compared to the bulk conductivity and frequency dependent dielectric measurements for a variety of ferroelectric polymer poly(vinylidene fluoride) (PVDF) copolymers and terpolymers. While some extra chemical groups introduced into the PVDF long chains increase the measured conductivities at room temperature and can cause significant changes in the dielectric properties, few changes are observed in the overall experimental molecular density of states. The exception appears to be the very short polymer chains. The significance of this comparison is that profound changes can occur in the overall dielectric and transport properties of this class of polymers and copolymers through molecular "dopant addition," without altering the overall density of states of the dominant PVDF polymer host. (C) 2009 American Institute of Physics.
\end{abstract}

[DOI: $10.1063 / 1.3204490$ ]

The ferroelectric polymer poly(vinylidene fluoride) (PVDF) as well as the associated copolymers with trifluoroethylene have high remnant polarization and coercive electric field, which makes these polymers suitable for nonvolatile random memory, as well as dielectric barrier layers. ${ }^{1-6}$ In making the copolymer PVDF with trifluoroethylene, $\mathrm{CH}_{2}-\mathrm{CF}_{2}: \mathrm{CHF}-\mathrm{CF}_{2}$, a reduction in the ferroelectric transition temperature is observed. ${ }^{7-10}$ Overall, it is clear as well that the introduction of chemical defects does have a profound effect on the dielectric and ferroelectric properties of the PVDF polymer backbone. If the long range all-trans conformation is broken by the introduction of defects, such as through irradiation by a high energy electron beam ${ }^{11,12}$ or by introduction of extra chlorofluoroethylene (CFE) or chlorotrifluoroethylene (CTFE) groups into poly(vinylidene fluoride-trifluoroethylene) $[\mathrm{P}(\mathrm{VDF}-\mathrm{TrFE})]$ copolymer, ${ }^{13,14}$ this polymer will behave like a ferroelectric relaxor ${ }^{11,15}$ with low remnant polarization and coercive field. In addition, PVDF homopolymers, when modified by bulky defects such as CTFE or hexafluoropropylene (HFP), may exhibit much reduced polarization hysteresis. ${ }^{16,17}$

The PVDF based polymers offer a family of compounds where the intramolecular dipole interactions may be systematically altered by the introduction of extra chemical groups (defects). One critical question is how the electronic structure of these polymers change as various extra chemical groups (defects) are introduced and how those changes are related to the intrinsic electronic properties, as opposed to more extrinsic properties such as interface states, formed at the metal electrode, that influence the performance of these polymers. ${ }^{18}$ This paper investigates the electronic structure of a series of PVDF based polymers, including the short

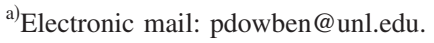

chain PVDF homopolymer and defect modified PVDF copolymers and terpolymer. Given that a wide range of bulk dielectric and ferroelectric properties are possible for this class of polymers, here is an opportunity to demonstrate that chemical doping that does not alter the overall electronic structure of the PVDF thin films significantly can still result in significant changes in the conductivity, polarization, and dielectric properties resulting from microscopic effects (local effects).

For the photoemission and inverse photoemission studies, poly(vinylidene fluoride with hexafluoropropylene) P(VDF-HFP; 96: $4 \mathrm{~mol} \%$ ) and poly(vinylidene fluoridechlorotrifluoroethylene) P(VDF-CTFE; 91: $9 \mathrm{~mol} \%$ ) copolymer and poly(vinylidene fluoride-trifluoroethylenechlorofluoroethylene) $\quad$ P(VDF-TrFE-CFE; 59.2: 33.6: $7.2 \mathrm{~mol} \%$ ) terpolymer films were prepared on Au coated silicon substrate by solution spin coating using DMF as solvent. The films have a thickness of about 10-20 nm and were subsequently annealed at $110^{\circ} \mathrm{C}$ for $\mathrm{P}$ (VDF-TrFE-CFE) and $130{ }^{\circ} \mathrm{C}$ for $\mathrm{P}(\mathrm{VDF}-\mathrm{HFP})$ and $\mathrm{P}(\mathrm{VDF}-\mathrm{CTFE})$ to improve the crystallinity. ${ }^{13,14}$ The short chain PVDF films were made through the resistant heating and evaporation of PVDF (Refs. 19 and 20) on Au substrate in a vacuum chamber, continuous to the vacuum chamber equipped with photoemission and inverse photoemission spectroscopies. The crystalline $\mathrm{P}(\mathrm{VDF}-\mathrm{TrFE}$; 65: 35) thin films were made on Au substrate by using the Langmuir-Blodgett technique, as described elsewhere. ${ }^{8}$ The thicknesses of PVDF polymer and crystalline P(VDF-TrFE; 65: 35) films were made so that no Au features show up in the molecular spectra in either photoemission or inverse photoemission.

For the electrical characterization measurements, a film thickness of around 10-20 $\mu \mathrm{m}$ was used. Aluminum electrodes of $40 \mathrm{~nm}$ were thermally deposited on both sides of the samples under high vacuum. The dielectric data were 
acquired by an $L C R$ meter (HP 4284A) equipped with a computer controlled temperature chamber. The $I-V$ curves were measured by HP $4140 \mathrm{~B}$ pA meter. ${ }^{13-18}$

Combined ultraviolet photoemission (UPS) and inverse photoemission (IPES) spectra were used to characterize the placement of both occupied and unoccupied states of the various PVDF polymer and copolymer thin films. Both the UPS and IPES spectra were taken in the same UHV chamber. In both photoemission and inverse photoemission measurements, the binding energies are referenced with respect to the Fermi edge of gold or tantalum, in intimate contact with the sample surface. The IPES were obtained by using variable energy electrons incident along the sample surface normal, while measuring the emitted photons at a fixed energy $(9.7 \mathrm{eV})$ using a Geiger-Müller detector with an instrumental linewidth of about $400 \mathrm{meV}^{20-28}$ The angle integrated photoemission (UPS) studies were carried out in the same vacuum system as IPES using a helium lamp at $h v=21.2 \mathrm{eV}$ (He I) and a Phi hemispherical electron analyzer with an angular acceptance of $\pm 10^{\circ}$ or more collecting photoemitted electrons along the sample surface normal. ${ }^{20,23,25,26,28}$

The semiempirical method NDO-PM3 (neglect of differential diatomic overlap, parametric model 3) model calculations based on Hartree-Fock formalism was used to produce occupied and unoccupied orbitals in order to simulate the UPS and IPES spectra. To compare the model calculations with experiment, we applied Gaussian envelopes of $1 \mathrm{eV}$ full width half maximum to each calculated molecular orbital (eigenvalue) to account for the solid state broadening in photoemission and then summing. These ground state PM3 model calculations, based on limited length molecular chain, are expected to differ from experiment, particularly as photoemission and inverse photoemission are the final state spectroscopies. As a practical matter, these semiempirical calculations require less ad hoc scaling than would be required for a comparison of $a b$ initio DFT calculations with experiment. ${ }^{29-31}$ In the comparison between the model calculations and experiment, no corrections were made for molecular interactions, matrix element effects and final state effects, but nonetheless, the comparison with experiment, although simplistic, is still often successful. ${ }^{20-23,28}$

Combined photoemission and inverse photoemission spectra of the polymer PVDF, the copolymer P(VDF-TrFE), poly(vinylidene fluoride-hexafluoropropylene) P(VDFHFP), poly(vinylidene fluoride-chlorotrifluoroethylene) $\mathrm{P}$ (VDF-CTFE), and the terpolymer poly(vinylidene fluoridetrifluoroethylene-chlorofluoroethylene) P(VDF-CTFE-CFE) are shown in Fig. 1, along with the model molecular orbital density of states for comparison. The simplistic model theory recovers most of the experimental features, especially for the highest occupied molecular orbitals (HOMOs).

The comparison of the combined photoemission and inverse photoemission spectra, for all of the thin films formed from different oligomer species, shows that most of the spectral features remain dominated by PVDF. The addition of extra chemical groups appears to have little influence on the overall spectra. The combined photoemission and inverse photoemission spectra for all of the different oligomer spe-

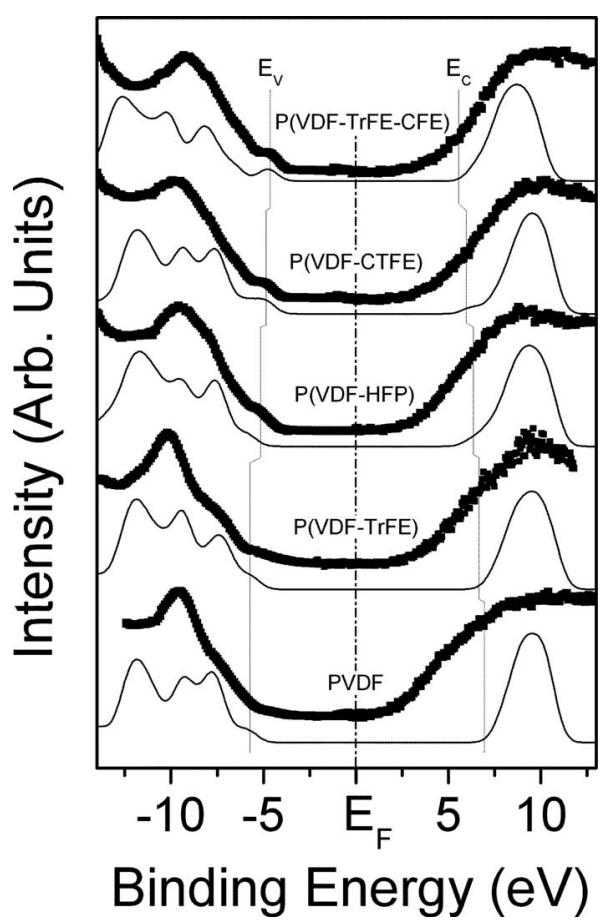

FIG. 1. The combined photoemission and inverse photoemission spectra (thick lines) of PVDF, P(VDF-TrFE), P(VDF-HFP), P(VDF-CTFE), and $\mathrm{P}(\mathrm{VDF}-\mathrm{TrFE}-\mathrm{CFE})$ taken at room temperature. Ground state model calculations of the density of states for a limited chain length molecule, uncorrected for matrix elements and final state effects, are shown for comparison as a thin line for each species. The expected model HOMO $\left(E_{V}\right)$ and LUMO $\left(E_{C}\right)$ of each molecule are marked by vertical bars; experiment is seen to differ.

cies remains very similar although the spectrum for evaporated PVDF exhibits the smallest HOMO-lowest unoccupied molecular orbital (LUMO) gap in experiment, due to the close proximity of the conduction band edge to the Fermi level (chemical potential). This is opposite to the simplest expectation (Fig. 1) where we would expect that PVDF would have the largest of all HOMO-LUMO gaps. This latter PVDF thin film differs from the other copolymer and terpolymer PVDF dominated thin films in that this is the one thin film with short PVDF chains. ${ }^{20}$ So decreasing the chain length may be the most profound manner which changes the overall electronic structure and suggests that the placement of the HOMO and LUMO are significantly affected by the defects resulting from the short chain lengths. The decrease in the HOMO to LUMO gap in Fig. 1 follows the tendency for decreasing symmetry in the polymer backbone as a result of chemical defect introduction. The introduction of the alien chemical groups (defects) into the PVDF long chains breaks the symmetry and long range dipole-dipole coupling. ${ }^{10,21} \mathrm{We}$ already have seen that in a similar vein, the ferroelectric to paraelectric transition results in a decrease in the HOMOLUMO gap and a tendency for the LUMO edge to shift toward the Fermi level in the paraelectric phase. ${ }^{23-26}$

Does the introduction of chemical dopants result in profound changes to the bulk electrical properties although few changes are seen in the electronic structure? The dielectric measurements of the copolymer P(VDF-HFP), P(VDFCTFE), and the terpolymer P(VDF-TrFE-CFE) are shown in Fig. 2. The dielectric constants decrease with the increasing 


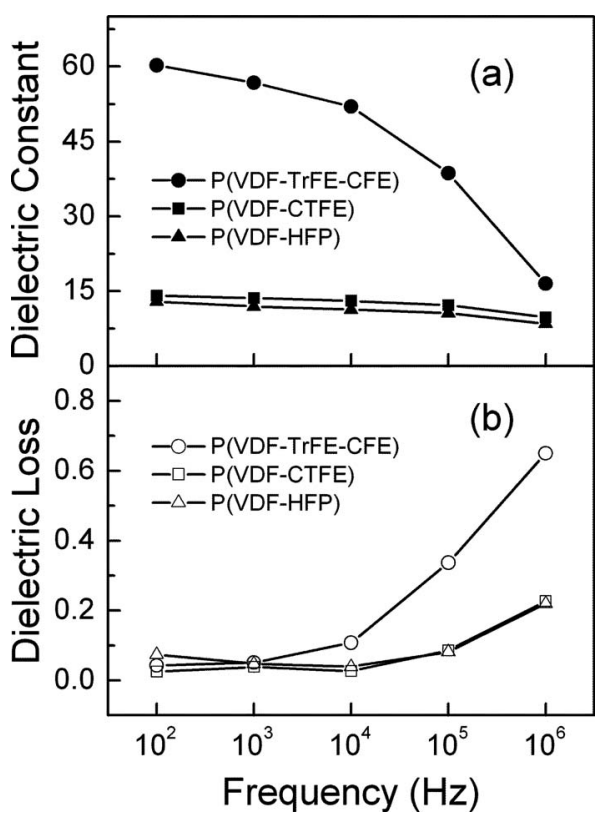

FIG. 2. Dielectric constants (a) and loss tangents (b) of P(VDF-HFP), $\mathrm{P}(\mathrm{VDF}-\mathrm{CTFE})$, and $\mathrm{P}(\mathrm{VDF}-\mathrm{TrFE}-\mathrm{CFE})$ at different frequencies of the external electrical field at room temperature.

external field frequency, while the loss tangents increase with frequency. This is fairly typical for this class of polar molecules. ${ }^{13-16}$ Although all three polymers follow the same trend for the dielectric constant and the loss, the terpolymer $\mathrm{P}$ (VDF-TrFE-CFE) is different from the other two copolymers. At the low frequency end $(100 \mathrm{~Hz})$, the P(VDF-TrFE$\mathrm{CFE}$ ) dielectric constant is four times larger than those of $\mathrm{P}(\mathrm{VDF}-\mathrm{HFP})$ and $\mathrm{P}$ (VDF-CTFE). Indeed, P(VDF-TrFECFE) only tends toward the values of $\mathrm{P}(\mathrm{VDF}-\mathrm{HFP})$ and $\mathrm{P}(\mathrm{VDF}-\mathrm{CTFE})$ at higher frequency $\left(10^{6} \mathrm{~Hz}\right)$, as shown in Fig. 2(a).

For the terpolymer P(VDF-TrFE-CFE) the room temperature loss tangent is three times lager than we observe for $\mathrm{P}\left(\right.$ VDF-HFP) and P(VDF-CTFE) at $10^{6} \mathrm{~Hz}$ and has almost same value as those of $\mathrm{P}(\mathrm{VDF}-\mathrm{HFP})$ and $\mathrm{P}(\mathrm{VDF}-\mathrm{CTFE})$ at lower frequency range $\left(10^{2}-10^{3} \mathrm{~Hz}\right)$, as shown in Fig. 2(b). The dielectric loss is related to the energy absorption due to the dipole relaxation in the material. At very high frequencies, the electronic resonant absorption will replace the dipole relaxation absorption and all three molecules should behave in a similar fashion.

The terpolymer $\mathrm{P}(\mathrm{VDF}-\mathrm{TrFE}-\mathrm{CFE})$ also has the highest static external electric field conductivity of the three copolymer studied, much larger than $\mathrm{P}(\mathrm{VDF}-\mathrm{HFP})$ and $\mathrm{P}(\mathrm{VDF}-$ CTFE), as shown in Fig. 3. The extra chemical groups are expected to provide additional hopping sites for the transport, resulting in an increase in the conductivity. At around $25 \mathrm{MV} / \mathrm{m}$ of external electric field, the current density of terpolymer $\mathrm{P}(\mathrm{VDF}-\mathrm{TrFE}-\mathrm{CFE})$ begins to saturate, while for copolymer $\mathrm{P}(\mathrm{VDF}-\mathrm{HFP})$ and $\mathrm{P}(\mathrm{VDF}-\mathrm{CTFE})$, their current densities keep increasing until $80 \mathrm{MV} / \mathrm{m}$ external electric field, although the latter remain more insulating than the terpolymer $\mathrm{P}(\mathrm{VDF}-\mathrm{TrFE}-\mathrm{CFE})$ thin films.

Overall, the introduction of extra chemical groups into PVDF thin films introduces defects in the all-trans conforma-

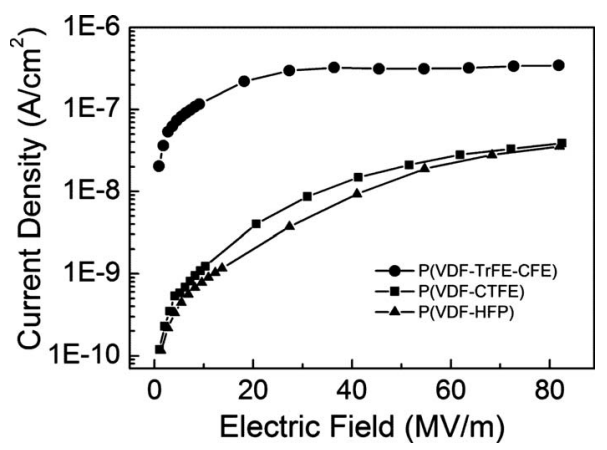

FIG. 3. Current density at the different strengths of the external static electric field for P(VDF-HFP), P(VDF-CTFE), and P(VDF-TrFE-CFE) at room temperature.

tion normally observed for this polymer system at room temperature. The defects break symmetry and long range intraand interchains dipole-dipole coupling. This in turn allows the individual chemical dipoles in the polymer chain to respond to external electric fields in a more facile manner. Without these defects, the strong dipole-dipole coupling tends to preserve the ferroelectric phase and hinder dipole rotation. The terpolymer P(VDF-TrFE-CFE), with the most defects, has the highest dielectric constant, dielectric loss and current density among these molecules. In this context, it should be noted that were all other electronic effects equal, the facility of dipole rotation and the greater conductivity should provide greater final state screening ${ }^{32,33}$ in the photoemission and inverse photoemission measurements of the terpolymer $\mathrm{P}(\mathrm{VDF}-\mathrm{TrFE}-\mathrm{CFE})$. This, in turn, should result in a smaller HOMO-LUMO gap, ${ }^{32}$ although, in fact, the smallest HOMO-LUMO gap is observed for the short chain PVDF thin films, primarily because of the shift in the LUMO toward the chemical potential as a result of a high defect density.

The energy level alignments of these various PVDF based polymer adlayers, with respect to the metal substrate, will be expected to be a little different from each other, and overall features should be very similar since the carbon backbone stays the same. In general, with some variation, this is, in fact, what we do observe. The dielectric and conductivity properties, together with the dipole response to an external electric field, differ widely for these polymers indicating that some of the dipole-dipole coupling is altered on the local scale. The introduction of defects (extra chemical groups) into PVDF molecular chain can break the all-trans conformation and produce the trans-gauche bonds in chains in $\mathrm{P}(\mathrm{VDF}-$ TrFE), P(VDF-HFP), P(VDF-CTFE), and P(VDF-TrFE$\mathrm{CFE}$ ) and could reduce the ferroelectric domain size.

\section{ACKNOWLEDGMENTS}

The work at University of Nebraska at Lincoln was supported by the National Science Foundation through Grant Nos. CHE-0415421 and CHE-0650453. The work at the Pennsylvania State University was supported by the Office of Navy Research through Grant No. N00014-05-1-0455 and the MURI under Grant No. N00014-05-1-0541.

\footnotetext{
${ }^{1}$ Ferroelectric Polymers, edited by H. S. Nalwa (Dekker, New York, 1995).
} 
${ }^{2}$ K. Tashiro, in Ferroelectric Polymers, edited by H. S. Nalwa (Dekker, New York, 1995), p. 63.

${ }^{3}$ The Applications of Ferroelectric Polymers, edited by T. T. Wang, J. M. Herbert, and A. M. Glass (Chapman and Hall, New York, 1988).

${ }^{4}$ A. J. Lovinger, Macromolecules 16, 1529 (1983).

${ }^{5}$ A. J. Lovinger, in Developments in Crystalline Polymers, edited by D. C. Basset (Elsevier Applied Science, London, 1982), Vol. 1.

${ }^{6}$ R. G. Kepler and R. A. Anderson, Adv. Phys. 41, 1 (1992).

${ }^{7}$ A. Bune, V. M. Fridkin, S. Ducharme, L. M. Blinov, S. P. Palto, A. Sorokin, S. G. Yudin, and A. Zlatkin, Nature (London) 391, 874 (1998).

${ }^{8}$ L. M. Blinov, V. M. Fridkin, S. P. Palto, A. V. Bune, P. A. Dowben, and S. Ducharme, Phys. Usp. 43, 243 (2000).

${ }^{9}$ T. Furukawa, Phase Transitions 18, 143 (1989).

${ }^{10}$ K. Tashiro, K. Takano, and M. Kobayashi, Ferroelectrics 57, 297 (1984).

${ }^{11}$ Q. M. Zhang, V. Bharti, and X. Zhao, Science 280, 2101 (1998).

${ }^{12}$ C. M. Othon, F. B. Bateman, and S. Ducharme, J. Appl. Phys. 98, 014106 (2005).

${ }^{13}$ H. Xu, Z.-Y. Cheng, D. Olson, T. Mai, Q. M. Zhang, and G. Kavarnos, Appl. Phys. Lett. 78, 2360 (2001).

${ }^{14}$ F. Xia, Z.-Y. Cheng, H. Xu, Q. M. Zhang, G. Kavarnos, R. Ting, G. Abdul-Sedat, and K. D. Belfield, Adv. Mater. (Weinheim, Ger.) 14, 1574 (2002).

${ }^{15}$ V. Bharti and Q. M. Zhang, Phys. Rev. B 63, 184103 (2001).

${ }^{16}$ B. Chu, X. Zhou, K. L. Ren, B. Neese, M. R. Lin, Q. Wang, F. Bauer, and Q. M. Zhang, Science 313, 334 (2006).

${ }^{17}$ X. Zhou, B. Chu, B. Neese, M. R. Lin, and Q. M. Zhang, IEEE Trans. Dielectr. Electr. Insul. 14, 1133 (2007).

${ }^{18}$ Q. Chen, B. Chu, X. Zhou, and Q. M. Zhang, Appl. Phys. Lett. 91, 062907 (2007).
${ }^{19}$ J. Choi, E. Morikawa, S. Ducharme, and P. A. Dowben, Mater. Lett. 59, 3599 (2005).

${ }^{20}$ J. Xiao and P. A. Dowben, J. Mater. Chem. 192172 (2009).

${ }^{21}$ J. Xiao, L. G. Rosa, M. Poulsen, D. Q. Feng, S. Reddy, J. M. Takacs, L. Cai, J. Zhang, S. Ducharme, and P. A. Dowben, J. Phys.: Condens. Matter 18, L155 (2006).

${ }^{22}$ J. Xiao, A. Sokolov, and P. A. Dowben, Appl. Phys. Lett. 90, 242907 (2007).

${ }^{23}$ P. A. Dowben, J. Xiao, B. Xu, A. Sokolov, and B. Doudin, Appl. Surf. Sci. 254, 4238 (2008).

${ }^{24}$ J. Choi, P. A. Dowben, S. Ducharme, V. M. Fridkin, S. P. Palto, N. Petukhova, and S. G. Yudin, Phys. Lett. A 249, 505 (1998).

${ }^{25}$ J. Choi, P. A. Dowben, S. Pebley, A. V. Bune, S. Ducharme, V. M. Fridkin, S. P. Palto, and N. Petukhova, Phys. Rev. Lett. 80, 1328 (1998).

${ }^{26}$ J. Choi, C. N. Borca, P. A. Dowben, A. Bune, M. Poulsen, S. Pebley, S. Adenwalla, S. Ducharme, L. Robertson, V. M. Fridkin, S. P. Palto, N. Petukhova, and S. G. Yudin, Phys. Rev. B 61, 5760 (2000).

${ }^{27}$ D. N. McIlroy, J. Zhang, P. A. Dowben, and D. Heskett, Mater. Sci. Eng., A 217-218, 64 (1996).

${ }^{28}$ J. Xiao, C. C. Ilie, N. Wu, K. Fukutani, and P. A. Dowben, Surf. Sci. 603, 513 (2009)

${ }^{29}$ I. N. Yakovkin and P. A. Dowben, Surf. Rev. Lett. 14, 481 (2007).

${ }^{30}$ A. M. Scheer and P. D. Burrow, J. Phys. Chem. B 110, 17751 (2006).

${ }^{31}$ A. M. Scheer, G. A. Gallup, and P. D. Burrow, Chem. Phys. Lett. 466, 131 (2008).

${ }^{32}$ J. E. Ortega, F. J. Himpsel, D. Li, and P. A. Dowben, Solid State Commun. 91, 807 (1994).

${ }^{33}$ P. A. Dowben, L. G. Rosa, C. C. Ilie, and J. Xiao, J. Electron Spectrosc. Relat. Phenom. (2008). 